ORIGINAL ARTICLE

\title{
A pilot study of the eccentric decline squat in the management of painful chronic patellar tendinopathy
}

\author{
C R Purdam, P Johnsson, H Alfredson, R Lorentzon, J L Cook, K M Khan
}

Br J Sports Med 2004;38:395-397. doi: 10.1136/bjsm.2003.000053

See end of article for authors' affiliations

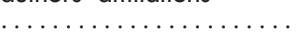

Correspondence to: Dr Cook, La Trobe University Musculoskeletal Research Centre, Victoria 3086, Australia; J.Cook@ latrobe.edu.au

Accepted 5 August 2003

\begin{abstract}
Objectives: This non-randomised pilot study investigated the effect of eccentric quadriceps training on 17 patients (22 tendons) with painful chronic patellar tendinopathy.

Methods: Two different eccentric exercise regimens were used by subjects with a long duration of pain with activity (more than six months). (a) Nine consecutive patients (10 tendons; eight men, one woman; mean age 22 years) performed eccentric exercise with the ankle joint in a standard (foot flat) position. (b) Eight patients ( 12 tendons; five men, three women; mean age 28 years) performed eccentric training standing on a $25^{\circ}$ decline board, designed to increase load on the knee extensor mechanism. The eccentric training was performed twice daily, with three sets of 15 repetitions, for 12 weeks. Primary outcome measures were (a) $100 \mathrm{~mm}$ visual analogue scale (VAS), where the subject recorded the amount of pain during activity, and (b) return to previous activity. Follow up was at 12 weeks, with a further limited follow up at 15 months.

Results: Good clinical results were obtained in the group who trained on the decline board, with six patients (nine tendons) returning to sport and showing a significantly reduced amount of pain over the 12 week period. Mean VAS scores fell from 74.2 to $28.5(p=0.004)$. At 15 months, four patients (five tendons) reported satisfactory results (mean VAS 26.2). In the standard squat group the results were poor, with only one athlete returning to previous activity. Mean VAS scores in this group were 79.0 at baseline and 72.3 at 12 weeks $(p=0.144)$.

Conclusion: In a small group of patients with patellar tendinopathy, eccentric squats on a decline board produced encouraging results in terms of pain reduction and return to function in the short term. Eccentric exercise using standard single leg squats in a similar sized group appeared to be a less effective form of rehabilitation in reducing pain and returning subjects to previous levels of activity.
\end{abstract}

$\mathrm{P}$ tellar tendinopathy is often a resistant and recurrent condition that primarily affects athletes in jumping sports. Treatment is initially conservative, and eccentric exercise is currently accepted as an important part of appropriate conservative therapy. ${ }^{1}$

The eccentric exercise commonly recommended for the patellar tendon is the squat. ${ }^{2}$ However, when a subject performs a squat, several mechanisms may unload the knee extensors and potentially reduce the eccentric load through the patellar tendon. Trunk flexion, side to side load sparing, and calf tension may all reduce the load placed on the knee. ${ }^{34}$

Passive and active calf tension have the potential to reduce demand on the knee extensors in the squat by limiting forward angulation of the tibia approaching the limit of ankle dorsiflexion. Purdam et $a l^{5}$ have suggested that this effect can be minimised, and load on the patellar tendon maximised, by performing a squat on a decline. As there has been no published research on the standard squat and the $25^{\circ}$ decline squat in the treatment of patellar tendinopathy, we investigated the effect of these techniques in the treatment of this condition.

The aim of this pilot study was to identify differences in pain reduction and recovery of function, using an eccentric single leg squat programme, on a flat surface and on a decline, in patients with patellar tendinopathy.

\section{MATERIALS AND METHODS Subjects}

Subjects referred to a university sports medicine clinic with the diagnosis of patellar tendinopathy were recruited to the study. All were examined by one orthopaedic surgeon (HA). The inclusion criteria were:
- pain in the proximal patellar tendon with increased load

- tenderness to palpation

- imaging changes at the proximal attachment of the tendon to the patella (ultrasonography or magnetic resonance imaging)

- having rested for more than three months without effect on the tendon pain

All patients had been treated with non-steroidal antiinflammatory drugs. One subject had had hydrocortisone injected as part of conservative treatment. Ethics approval was obtained from the University of Umeå, Sweden and all subjects provided informed consent.

\section{Intervention}

Subjects in this pilot study were not randomised because of the later development and inclusion of the decline exercise technique. Thus, the first nine consecutive subjects presenting for evaluation and agreeing to participate in the study were prescribed eccentric squats with the ankle joint in a standard position (fig 1). The next eight subjects were prescribed eccentric squats on a $25^{\circ}$ decline board (fig 2).

Both groups undertook an exercise programme twice daily, consisting of three sets of 15 repetitions on a single leg, for 12 weeks. Those subjects with bilateral tendinopathy completed the programme separately for each leg. Subjects were instructed to complete the exercises with the trunk upright. Both groups were instructed to perform the exercise by slowly flexing the knee to $90^{\circ}$ of flexion, and, as far as possible, to perform eccentric loading of the quadriceps muscles only and to return to the starting position using the non-injured side. 


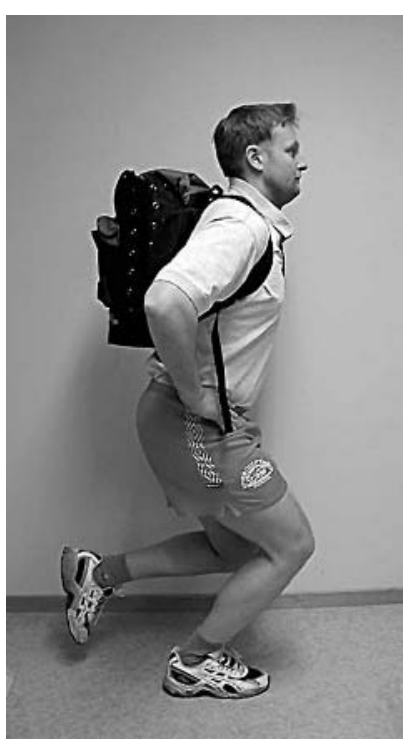

Figure 1 Standard squat technique.

If they had bilateral tendinopathy, the arms and both legs were used to return to the starting position.

Subjects were asked to increase the load with weights in a backpack once the exercise could be completed without pain. Load was increased so that exercises were always performed with some pain or discomfort.

Subjects were not allowed to continue their competitive sporting activity during the first eight weeks of the trial period. After four weeks of the eccentric training regimen, they were allowed to complement it with slow jogging on flat ground, cycling, and water activities, if these could be performed without sharp pain in the patellar tendon. After eight weeks the patients were allowed to gradually return to previous activity.

\section{Outcome measures}

Visual analogue scale (100 point) (VAS) scores were recorded at baseline and at completion of the 12 weeks, to record the amount of pain during patellar tendon loading activity-that is, volleyball players during volleyball, soccer players during soccer, etc.

A further follow up was conducted at 15 months in the decline squat group of all tendons not managed surgically.

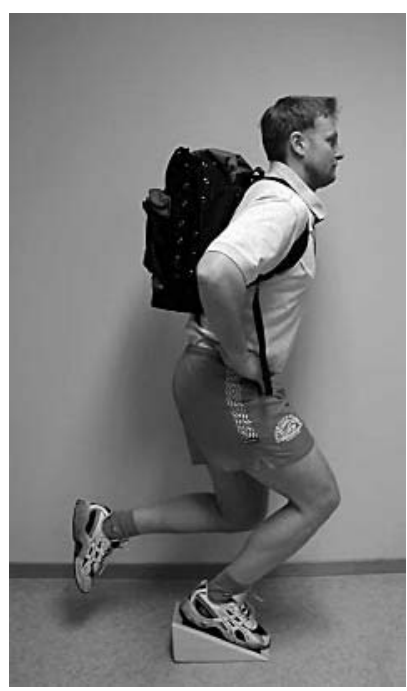

Figure 2 Decline squat technique.

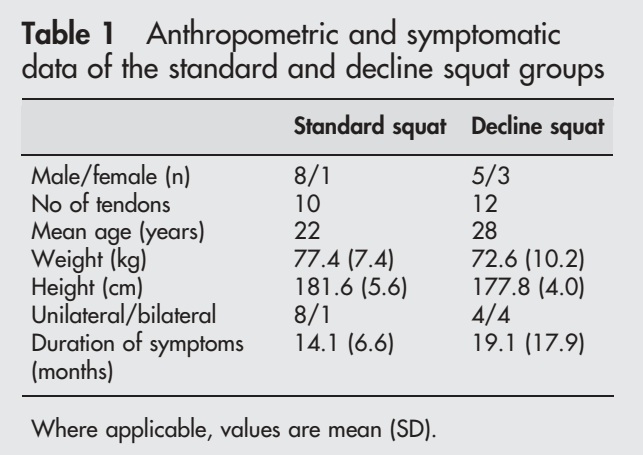

Patient ability to return to previous (pre-injury) activity level was also recorded.

\section{Statistical analysis}

Subject characteristics of both groups were tabulated using descriptive statistics. VAS scores recorded before and after the eccentric programme were analysed for each group using a Wilcoxon matched pairs test (Statistica Release 6, 2002; Statsoft Inc). Two tailed significance was set at $\mathrm{p}<0.05$. Because of limitations in study design, no between group comparison was performed.

\section{RESULTS}

\section{Subjects}

Seventeen subjects completed the intervention. At baseline, there were no significant differences in height, weight, and duration of symptoms between groups (table 1). All subjects were competitive in a range of sports before presentation (table 2).

\section{Visual analogue scale}

Table 3 shows descriptive results. There was a significant ( $p$ $=0.004)$ decrease in the amount of pain during activity (mean VAS score reduced from 74.2 to 28.5 ) in the decline squat group, between the measurements before and after the 12 week eccentric training regimen. No significant change was found in the standard squat group for the same period of training (mean VAS scores 79.0 at baseline and 72.3 at 12 weeks, $\mathrm{p}=0.144)$.

\section{Return to activity}

Six subjects (nine tendons) in the decline group had returned to pre-injury activity levels in their sports. The remaining subjects had not been able to return to their previous activity level and were referred for surgical treatment. In the standard squat group, only one subject (one tendon) had been able to return to previous activity level. The remaining eight subjects (nine tendons) were also referred for surgical treatment.

\begin{tabular}{|c|c|c|}
\hline & Normal squat $(n=9)$ & Decline squat $(n=8)$ \\
\hline Floorball & 4 & 0 \\
\hline Soccer & 2 & 1 \\
\hline Ice hockey & 1 & 1 \\
\hline Running & 0 & 2 \\
\hline High jump & 1 & 1 \\
\hline Volleyball & 0 & 3 \\
\hline Skiing & 1 & 0 \\
\hline
\end{tabular}


Table 3 Mean visual analogue scores (VAS) for standard and decline squat groups at baseline and follow up

\begin{tabular}{|c|c|c|c|c|c|}
\hline & \multicolumn{2}{|c|}{ Standard squat } & \multicolumn{3}{|c|}{ Decline squat } \\
\hline & Baseline & $\begin{array}{l}12 \text { week } \\
\text { follow up }\end{array}$ & Baseline & $\begin{array}{l}12 \text { week } \\
\text { follow up }\end{array}$ & $\begin{array}{l}15 \text { month } \\
\text { follow up }\end{array}$ \\
\hline VAS pain score & $79.0(7.0)$ & $72.3(19.14)$ & $74.2(11.6)$ & $28.5(29.4)^{*}$ & $26.2(25.4)$ \\
\hline Number of tendons & 10 & 10 & 12 & 12 & 5 \\
\hline
\end{tabular}

VAS scores are mean (SD)

*significant differently from baseline $(p=0.004)$.

\section{Take home message}

Patellar tendinopathy may be treated more effectively by application of eccentric exercise with the use of a decline board. The decline probably enables better isolation of the knee extensor mechanism in squat exercises.

\section{5 month follow up}

The nine tendons in the decline group above were followed up at 15 months. Four of the six subjects (five of nine tendons) described in the decline group above were still active at pre-injury levels. One subject (two tendons) reported no symptoms in his patellar tendons, yet was unable to continue his sport because of the development of bilateral patellofemoral pain syndrome after jump training. Colour Doppler ultrasonography of the patellar tendons showed them to be normal. A further subject (two tendons) had a relapse of significant patellar tendon pain (mean VAS score 68) and had tried an alternative treatment within the follow up period. Colour Doppler ultrasonography in this case showed changes consistent with patellar tendinopathy.

The overall mean (SD) of the VAS scores at 15 months for the five remaining tendons in the decline group was 26.2 (25.4).

\section{DISCUSSION}

Few studies have investigated the effect of conservative treatment on patellar tendinopathy. ${ }^{2}{ }^{6}$ Curwin and Stanish ${ }^{7}$ noted that patellar tendinitis responded less well to their eccentric programme than intervention in several other tendons. We and others have also noted this in our clinical practices. Thus, we sought modes by which eccentric exercise may have greater efficacy when applied to patellar tendinopathy. This pilot study is the first report of the effect of eccentric exercise performed as a decline squat in the treatment of painful patellar tendinopathy. However, it must be clearly recognised that, by its nature, it is non-randomised and contains low numbers.

In this study, subjects performing the eccentric squat on the decline board showed good clinical results, with a decreased amount of pain during activity (VAS score) and a return to previous activity level. Most subjects who performed the standard squat had unsatisfactory clinical results over the 12 week treatment period. We speculate that the effectiveness of the decline squat may be the result of the decline board reducing calf muscle tension, allowing better isolation of the knee extensor mechanism.

We consider that from the results of this small pilot study, further clinical studies are warranted to test the efficacy of the exercise. Such studies should use larger numbers, a randomised study design, and more specific outcome measures such as the VISA. ${ }^{8}$ A similar follow up period would also be appropriate to better capture the effect of these exercise programmes on sustained return to sporting activity. Biomechanical studies comparing the relative loads on the knee extensor mechanism with a standard and decline squat would also be of benefit.

\section{Limitations of this study}

This study did not use a randomised design, as subjects who presented first were given squat exercise without a decline board, because at that time the decline squat had not been developed. However, we were cautious not to change any other aspects of the study design for the decline board intervention. The subject numbers of the study are small, particularly at the 15 month follow up. Outcome measures should be extended to include a more specific outcome measure such as the VISA scoring system developed specifically for patellar tendinopathy. ${ }^{8}$

\section{CONCLUSION}

The results of this pilot study indicate that an eccentric exercise programme using the decline squat may have promise for subjects with chronically painful patellar tendinopathy. However, as the number of patients included was small, the data should be interpreted cautiously. Randomised studies comparing different models of squat exercise are needed.

\section{Authors' affiliations}

C R Purdam, Department of Physical Therapies, Australian Institute of Sport, PO Box 176, Belconnen 2616, ACT, Australia

$\mathrm{P}$ Jonsson, $\mathrm{H}$ Alfredson, $\mathrm{R}$ Lorentzon, Department of Surgical and Perioperative Sciences, Sports Medicine Unit, University of Umeå, Sweden

J L Cook, La Trobe University Musculoskeletal Research Centre, Victoria, Australia

K M Khan, Department of Family Practice and School of Human Kinetics, University of British Columbia, Canada

\section{REFERENCES}

1 Cook JL, Khan KM, Purdam CR. Conservative treatment of patellar tendinopathy. Physical Therapy in Sport 2001;2:54-65.

2 Cannell $\mathrm{L}$, Taunton JE, Clement DB, et al. A randomised clinical trial of the efficacy of drop squats or leg extension/leg curl exercises to treat clinically diagnosed jumper's knee in athletes: pilot study. Br J Sports Med $2001 ; 35: 60-4$.

3 Mclaughlin T, Lardner T, Dillman C. Kinetics of the parallel squat. Res $Q$ 1978;49:176-89.

4 Wilk K, Escamilla R, Fleisig G, et al. A comparison of tibiofemoral joint forces and electromyography activity during open and closed kinetic chain exercises. Am J Sports Med 1996;24:518-27.

5 Purdam C, Cook J, Hopper D, et al. Discriminative ability of functional loading tests for adolescent jumper's knee. Physical Therapy in Sport 2003;4:3-9.

6 Jenson K, Di Fabio RP. Evaluation of eccentric exercise in treatment of patellar tendinitis. Phys Ther 1989;69:211-16.

7 Curwin S, Stanish WD. Tendinitis: its etiology and treatment. Lexington: Collamore Press, 1984.

8 Visentini PJ, Khan KM, Cook JL, et al. The VISA score: an index of the severity of jumper's knee (patellar tendinosis). J Sci Med Sport 1998;1:22-8. 